\title{
The power of analogies
}

\author{
Black holes, gravitational lenses, turbulence, chaotic flow and rogue waves are just a few examples of \\ complex physical phenomena that can be conveniently modelled using photonics.
}

As one would expect, Nature Photonics strives to publish findings that represent important conceptual insights or technological advances in the field of optics. However, we have also been publishing studies in which photonics proves to be a useful platform for mimicking and gaining a better understanding of other physical systems, such as those found in astrophysics and hydrodynamics.

Photonics is particularly suitable for such studies as it can often allow largescale, highly inaccessible and rare events in the natural world to be recreated on demand and explored on a lab bench using modest equipment. For example, optical metamaterials can now be engineered to behave in an analogous fashion to black holes $^{1}$ and gravitational lensing has recently been mimicked on an optical polymer chip ${ }^{2}$. Furthermore, fibre optics is being extensively used to generate and explore complex nonlinear wave phenomena found in fluid flows, including turbulence ${ }^{3}$, rogue waves ${ }^{4}$, modulational instability ${ }^{5}$, solitons ${ }^{6}$ and chaos ${ }^{7}$.

"Research in optics is interesting in its own right, but it also has the potential to model other systems on a table-top scale and under ambient conditions," comments Daniel Solli, an expert in nonlinear systems from the University of California at Los Angeles in the USA and the University of Göttingen in Germany. "The timescales of optical experiments are also often short, and by employing devices such as megahertz-rate mode-locked lasers it is possible accumulate large data sets in a short period of time."

Solli says that photonics experiments have helped explain the origin of rogue waves large freak waves that occasionally occur in the ocean. "We've narrowed it down to a set of specific input noise conditions, and made measurements that would never have been possible in hydrodynamics."

Solli points out another benefit of photonics - the very high level of control it offers. For example, it is possible to tailor the frequency, shape, strength, duration and timing of light pulses. Optical measurement technologies are also well developed in optics, with a plethora of high-performance instruments available. They include ultrafast, highly sensitive detectors and high-resolution spectrum analysers, which make it possible to capture single-shot dynamics and collect a wealth of information.

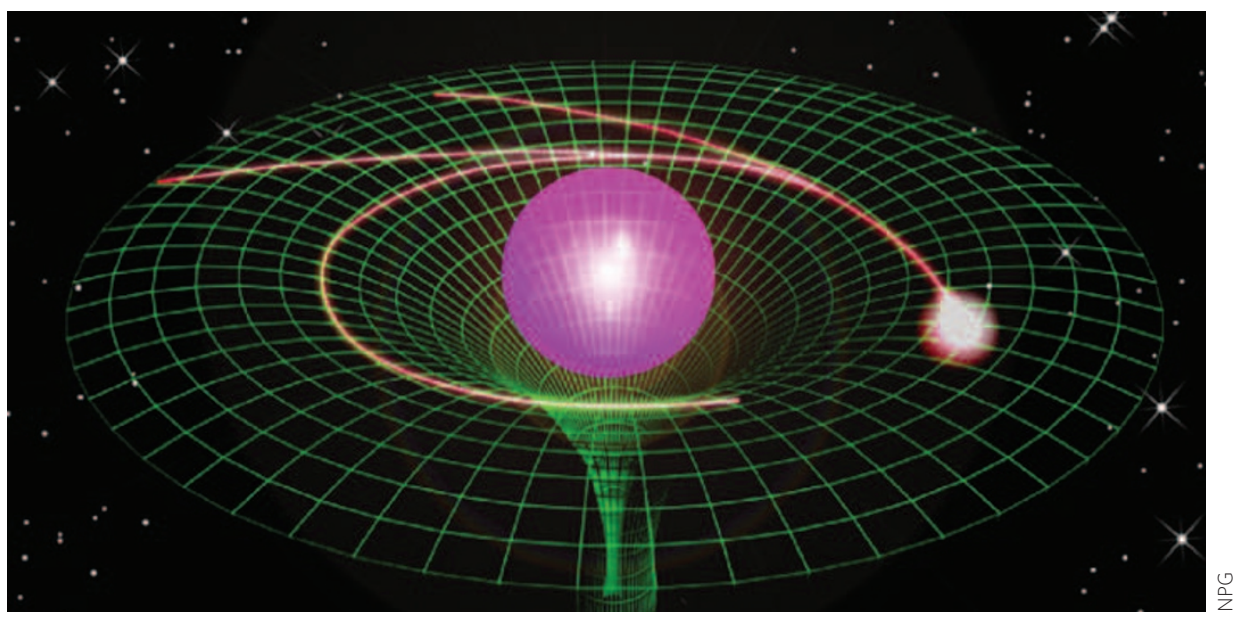

Solli and co-workers are currently exploring modulational instability, a nonlinear phenomenon that is ubiquitous in nature and can influence the shapes of sand ripples, cloud formations and heart rhythms and play an important role in the generation of broadband optical supercontinuua ${ }^{8}$.

Dentcho Genov at Louisiana Tech University in the USA is another researcher who has been using photonics to model physical systems. He uses unique optical structures (in particular, custom-designed metamaterials and microstructured waveguides) to mimic astrophysical phenomena such as celestial mechanics, Einstein's field equations, gravitational lensing and the event horizons of black holes.

Genov explains that an equivalence exists between the motion of masses and light in curved space-time and light propagation in a locally engineered optical material. An example of this optical-mechanical analogy is the similarity between Fermat's principle in optics for light ray propagation and the principle of least action for the motion of a particle.

"It's all about creating an inhomogeneous optical medium where the refractive index is carefully engineered to reproduce the desired phenomena optically," explained Genov. "It's not possible to actually visit a black hole and analyse the behaviour of light in close proximity to it, so if we can mimic and model it in the lab, that's a very powerful and useful tool."

He notes that the use of coordinate transformation theory for designing artificial metamaterials with specific optical properties, and the increasing sophistication of nanofabrication techniques are key enablers for realizing this analogy in practice. "We have known for a long time the dynamic equations that allow photons to represent mechanical particles, but we couldn't make the required refractive index profile. We can now do that with metamaterials."

Genov and colleagues have recently reported an optical analogue of a gravitational lens. They realized this by using an integrated optical circuit in which an optical waveguide surrounds a microsphere attached to a polymer chip. The microsphere bends light propagating on the planar chip in the same manner that a star acting as a gravitational lens bends light ${ }^{2}$.

This small selection of examples illustrates the exciting potential of photonics research to explore fields lying beyond the realm of optics.

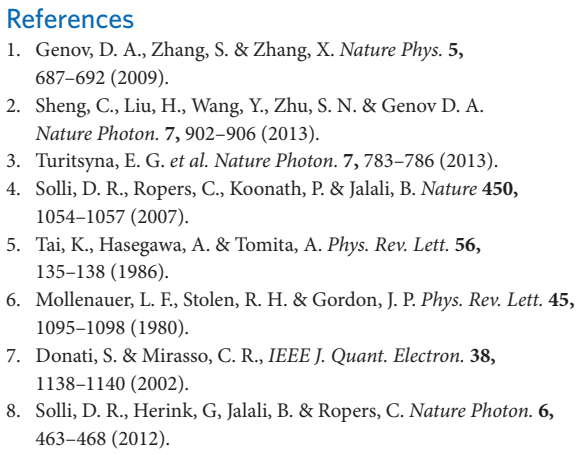

